\title{
The Inertial Energy Control Strategy of the Cascade H-Bridge Module applied in Photovoltaic System
}

\author{
Zhao Chenghan ${ }^{1, *}$, Li Xiang ${ }^{2}$, Xie $\mathrm{Da}^{1}$, and Pan Mingjie ${ }^{1}$ \\ ${ }^{1}$ Shanghai Jiaotong University, Department of Electrical Engineering, 200240 Shanghai, China \\ ${ }^{2}$ Shanghai Dianji University, Department of Electrical Engineering, 201306 Shanghai, China
}

\begin{abstract}
This paper put forward the inertial control strategy based on the control of charge and discharge of supercapacitor and the trigger angle of $\mathrm{H}$-bridge which integrates photovoltaic (PV) system. The whole cascade H-bridge structure is composed of traditional photovoltaic module, Boost/Buck circuit, supercapacitor (SC), and H-bridge circuit. First, the charging state and the inertial energy power support of the SC are analysed. Then the control strategy of the cascade H-bridge is proposed. To verify the efficiency of the control strategy, a cascade chain consists of $12 \mathrm{H}$-bridge unit was built in EMTP simulation software. Finally the simulation results prove that the newly developed SC-integrated cascade module can do inertial energy support well, which is important to the coordination between the power system and solar energy.
\end{abstract}

\section{Introduction}

Photovoltaic power fluctuations caused by environmental factor are fairly violent. At the same time, the grid inverter's fast dynamic response and its small inertial energy makes itself lack of the ability of frequency modulation and voltage regulation of power grids. Besides, high frequency electronic switch composed of various kinds of inverters are generally contain a certain composition of harmonics which affect the power grid power quality [1]. Therefore, photovoltaic is not a stable source of energy. Secondly, at present, most photovoltaic power stations are gridconnected by photovoltaic arrays composed of series and parallel photovoltaic modules. Those PV stations can only adjust the maximum power of photovoltaic arrays by using the maximum power point tracking (MPPT), but cannot guarantee the maximum power operation of every photovoltaic module [2], resulting in a certain amount of energy waste. Generally speaking, the current solar photovoltaic power generation is not grid-friendly.

In order to solve the above problems, industry and academia have carried out a lot of research work. First, the virtual synchronous generator (VSG) technology was studied [3]-[8]. A cascade photovoltaic power generation system with the characteristics of synchronous motor was designed [5]-[8], so that it has the inertia and damping characteristics of synchronous generator and can suppress the random power fluctuation of photovoltaic. The above research hopes to make the solar photovoltaic power station equivalent to a virtual synchronous machine through the control algorithm of the inverter, so that it has the ability to support the dispatching for frequency modulation and voltage regulation.
The authors proposed that three-level circuit can be used in solar photovoltaic inverter, which greatly reduced the harmonic content of inverter and greatly improved the power quality [9]. Some papers only proposed the DC side voltage balance control strategy for cascade H-bridge photovoltaic grid-connected inverter, without considering the situation that photovoltaic modules would be mismatched or completely obscured under the influence of the environment [10]-[12]. Therefore, in this paper we propose a new structure of photovoltaic modules based on cascade H-bridge, and its main contributions are as follows:

1) A kind of chain-cascading topology with inertial energy link is proposed, which is applied to solar photovoltaic power generation and provides the basis of grid-friendly control for solar photovoltaic power generation.

2) The MPPT algorithm of the underlying voltage regulator circuit and the inertial energy control cascade H-bridge photovoltaic module control algorithm are proposed, which realize the grid friendliness.

3)The chain sorting algorithm without communication and the $n-k$ lack-chained control technology is proposed to guarantee the reliability improvement of solar photovoltaic power generation.

The composition of this paper includes the following parts: Part II introduces the cascade H-bridge photovoltaic module topology and three-phase grid topology; Part III introduces the control strategy of cascade $\mathrm{H}$ bridge photovoltaic module; Part IV builds a 12 H-bridge simulation model and carries out gridconnection experiment to verify the correctness of the above theory. The conclusion is presented in part $\mathrm{V}$. 


\section{Cascade H-bridge PV Module Topology}

Fig. 1 shows the topology of cascade H-bridge photovoltaic module, which is mainly composed of traditional photovoltaic module, Boost/Buck circuit (referred to as B/B circuit here), supercapacitor and $\mathrm{H}$ bridge circuit. Among them, the photovoltaic module is composed of 60 photovoltaic cell monomers in series, and every 20 reverse parallel a diode, to prevent local shelter or damage, causing the whole photovoltaic module to stop working.

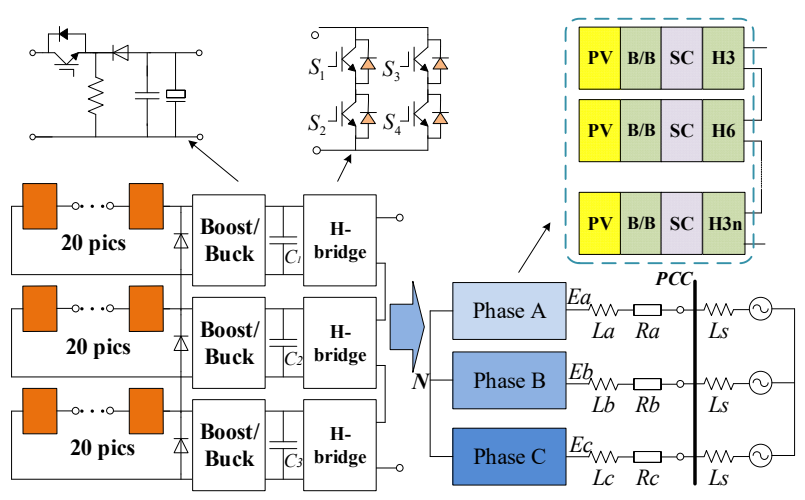

Fig. 1. Topology structure of cascade H-bridge PV modules

The combination of $\mathrm{B} / \mathrm{B}$ circuits and supercapacitors (SC), on the one hand, can be released or by super capacitor stored energy to balance the input voltage of the H-bridge circuit. On the other hand they can achieve frequency and voltage regulating functions through certain control algorithm which is similar to the ones of synchronous generator inertia control. So the power grid stability will get improved and the penetration rate of new energy will get raised. The cascade H-bridge circuit is composed of three H-bridge modules. The required step wave is generated by controlling the on-off of the power switch tube of each H-bridge module. Finally, the step wave is superposed to generate the required $\mathrm{AC}$ waveform.

When $\mathrm{n}$ of the above H-bridge circuits, which has 3 $\mathrm{H}$-bridges cascade inside each photovoltaic module, are cascade, the chained photovoltaic modules will contain $3 n$ H-bridges. If the output voltage of $n$ photovoltaic modules after cascading is equal to the grid voltage, it can be directly connected to the $220 \mathrm{~V}$ AC grid, eliminating the disadvantages of the traditional connection method requiring $\mathrm{DC} / \mathrm{AC}$ converter and simplifying the control complexity.

\section{Control Strategy for Cascade H-bridge PV Module}

\subsection{Supercapacitor Inertial Energy Support}

On average, the output voltage of each series of photovoltaic modules can be maintained at $12 \mathrm{~V} \sim 13.5 \mathrm{~V}$. Excessive voltage increases the reactive power of the system, resulting in energy waste. Therefore, the maximum voltage at both ends of the supercapacitor is $13.5 \mathrm{~V}$. However, in order to connect the photovoltaic system with the grid stably, its output voltage must be consistent with the grid voltage, and the error rate cannot exceed $5 \%$. Therefore, a certain threshold value must be set for the supercapacitor.

$$
U_{c \min }=\frac{\sqrt{2} U_{r m s}}{n}
$$

where, $U_{r m s}$ is the effective value of single-phase voltage in the power network, and $n$ is the number of $\mathrm{H}$ bridge cascades. $U_{c \min }$ is the minimum value of the voltage threshold for SC.

Figure 2 shows the charging and discharging range of the SC. $U_{\text {cpref }}$ is the reference voltage of the SC, and its value is $\frac{U_{c \max }+U_{c \min }}{2}$. When the voltage of the $\mathrm{SC}$ is bigger than $U_{c \max }$ or less than $U_{c \min }$, the circuit stops working. When $U_{\text {cpref }}<U_{c}<U_{c \max }$, the circuit operates in Buck mode; When $U_{c \text { min }}<U_{c}<U_{\text {cpref }}$, the circuit operates in Boost mode. The conversion between Buck and Boost mode makes the output voltage of the SC stable at the reference voltage.

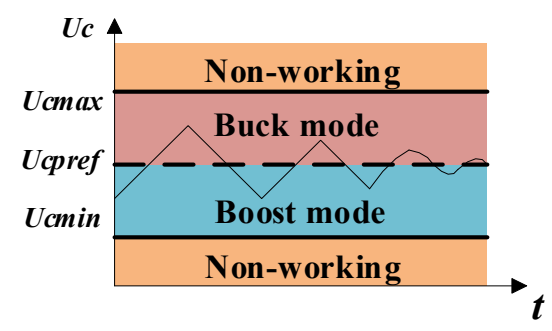

Fig. 2. Supercapacitor working range

In order to make the cascade H-bridge photovoltaic modules have the ability of inertial support and spontaneously responding to the system frequency changes, this paper proposes a virtual synchronous control strategy based on the inertial characteristics of synchronous generators.

Suppose the initial voltage at both ends of the supercapacitor is $U_{0}$, then the initial storage energy $E_{0}$ is:

$$
E_{0}=\frac{1}{2} C U_{0}^{2}
$$

However, due to the influence of environmental factors on photovoltaic modules, the voltage at both ends of the supercapacitor at time $t$ changes to $U(t)$, and the energy change $\Delta E$ of the supercapacitor is:

$$
\Delta E(t)=\frac{1}{2} C\left(U_{0}^{2}-(V(t))^{2}\right)
$$

Then the output power of the PV system at time $t$ is:

$$
P_{C}(t)=\frac{d \Delta E(t)}{d t}=-C U(t) \frac{d U(t)}{d t}
$$

Since the voltage $U(t)$ is the output voltage of $\mathrm{B} / \mathrm{B}$ circuit, it can be expressed as: 


$$
U(t)=\frac{D}{1-D} U_{i}(t)
$$

where, $U_{i}(t)$ is the output voltage of each series of photovoltaic modules at time $t$, that is, the input voltage of $\mathrm{B} / \mathrm{B}$ circuit; $D$ is the duty cycle of the $\mathrm{B} / \mathrm{B}$ circuit. When $0<D<0.5$, the circuit operates in Boost mode. When $0.5<D<1$, it operates in Buck mode.

Replace the $U(t)$ in equation (4) using equation (5):

$$
\begin{aligned}
P_{C}(t) & =-C \frac{D}{1-D} U_{i}(t) \frac{d\left(\frac{D}{1-D}\right) U(t)}{d t} \\
& =-C\left(\frac{D}{1-D}\right)^{2} U_{i}(t) \frac{d U_{i}(t)}{d t}
\end{aligned}
$$

Equation (6) is the supercapacitor inertial energy support power when the output voltage of photovoltaic modules fluctuates, which can suppress the impact of random fluctuations of photovoltaic output power on the power grid.

\subsection{Cascade $\mathrm{H}$-bridge control strategy}

The H-bridge circuit converts DC into AC. Each Hbridge has two switch tubes on the upper and lower arm of the bridge. There are $2 * 2=4$ conduction conditions in total, and three output voltage levels: $+\mathrm{E}, 0$ and $-\mathrm{E}$. If $n$ $\mathrm{H}$-bridge photovoltaic modules are cascade, then there are $3 n$ H-bridge chains, which can output $6 n+1$ levels in total. Therefore, the amplitude of the AC output voltage of the cascade H-bridge can be obtained according to the number of effective working chains of the H-bridge in the circuit. Different output waveforms can be obtained by only controlling the trigger angle of the switch tube of each H-bridge unit.

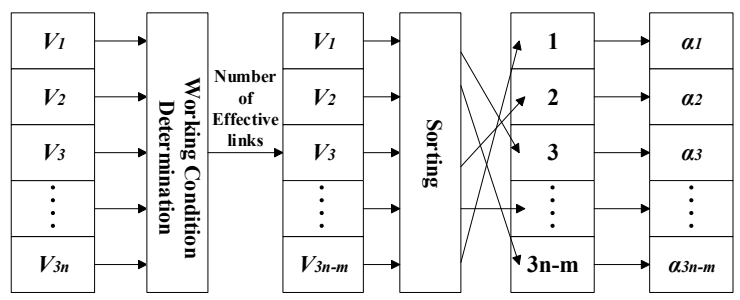

Fig. 3. Cascade H-bridge control strategy

Figure 3 shows the cascade H-bridge control strategy. First, each H-bridge is numbered according to the location of the chain. Then, the DC voltage detection circuit is used to collect $3 n$ DC side voltages of $\mathrm{H}$ bridge and upload them to the control unit. The control unit judges the working state of each link according to the received voltage and obtains the number of effective links. Then it sorts the voltage data received according to the current number of effective link and calculates the trigger Angle of each link switch tube like placing the chain with the highest voltage as number one and the next one as number two etc. Finally, the control unit will issue the total number of links and the sorting number of the link to the controller for each link.
Tab. 1. 12 H-bridge and 11H-bridge trigger Angle table

\begin{tabular}{ccccc}
\hline \multirow{2}{*}{ Number } & \multicolumn{2}{c}{12 H-bridge } & \multicolumn{2}{c}{11 H-bridge } \\
\cline { 2 - 5 } & $\begin{array}{c}\text { Conduction } \\
\text { Angle }\end{array}$ & $\begin{array}{c}\text { Turn-off } \\
\text { Angle }\end{array}$ & $\begin{array}{c}\text { Conduction } \\
\text { Angle }\end{array}$ & $\begin{array}{c}\text { Turn-off } \\
\text { Angle }\end{array}$ \\
\hline 1 & 0.039 & 179.961 & 0.038 & 179.962 \\
2 & 10.342 & 169.658 & 12.233 & 167.767 \\
3 & 10.360 & 169.640 & 25.325 & 154.675 \\
4 & 18.130 & 161.870 & 38.574 & 141.426 \\
5 & 22.336 & 157.664 & 39.712 & 140.288 \\
6 & 28.210 & 151.790 & 51.387 & 128.613 \\
7 & 33.573 & 146.427 & 63.139 & 116.861 \\
8 & 40.138 & 139.862 & 73.237 & 106.763 \\
9 & 46.601 & 133.399 & 81.248 & 98.752 \\
10 & 55.852 & 124.148 & 86.807 & 93.193 \\
11 & 67.577 & 112.423 & 89.641 & 90.359 \\
12 & 79.279 & 100.721 & - & - \\
\hline
\end{tabular}

The working state of each link can be judged according to the DC side voltage of $\mathrm{H}$-bridge unit. When the DC-side voltage is in the optimal state or still within the range of the maximum threshold or minimum threshold voltage even if it is affected by the environment changes, it is an effective link. When the PV module is completely shaded and has little power output or exceeds the threshold range, it is not an effective link.

The trigger angle of each chain switch can be calculated by the equivalence principle of impulse area. This paper takes $12 \mathrm{H}$-bridge cascading as an example to calculate the trigger angles of $12 \mathrm{H}$-bridge and $11 \mathrm{H}$ bridge respectively. The results are shown as Table 1.

\section{Simulations and Analysis}

In order to verify the effectiveness of the cascade $\mathrm{H}$ bridge control strategy, a model consists of $12 \mathrm{H}$-bridge unit was built in EMTP simulation software. Fig. 4 shows the DC side output voltage of $12 \mathrm{H}$-bridge units. Due to the support of DC side voltage inertia, the voltage of each H-bridge is basically stable at about $12 \mathrm{~V}$.

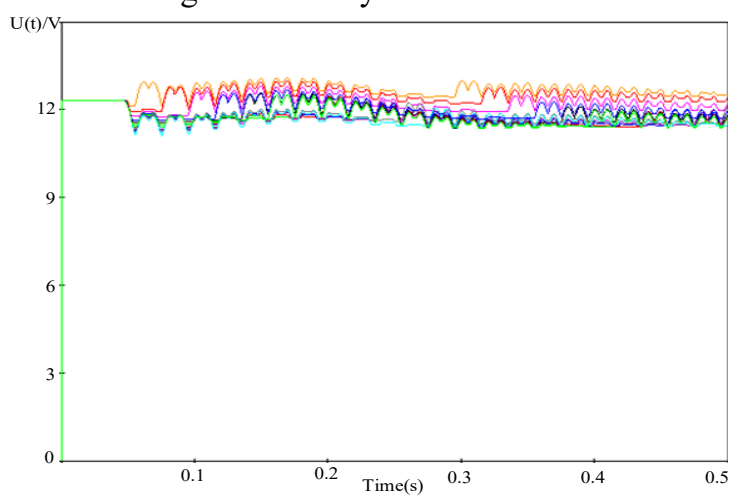

Fig. 4. DC side voltage of $\mathrm{H}$ bridge

Fig. 5 shows the single-phased output voltage waveform during operation of $12 \mathrm{H}$-bridge chain and 11 $\mathrm{H}$-bridge chain. As can be seen from the figure, the output waveform is a sinusoidal wave fitted by step wave. It can be seen from the voltage waveform: in the 
case of $12 \mathrm{H}$-bridge cascade, the output waveform is superposed by 25 levels and closely approaches to sinusoidal wave.

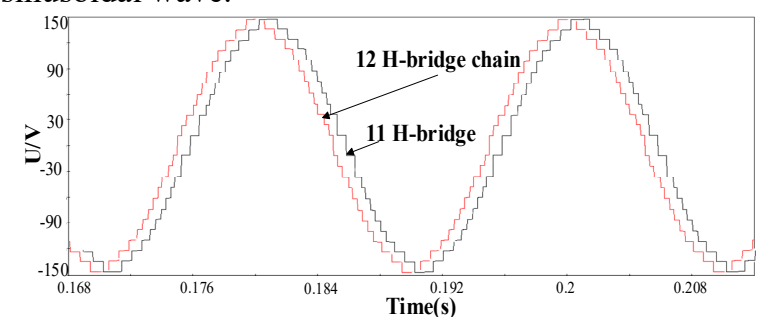

Fig. 5. H-bridge output voltage waveform

Fig. 6 shows the active and reactive power curves of the cascade $\mathrm{H}$-bridge circuit. When the output voltage amplitude of the cascade H-bridge is constant, the power output can be changed by adjusting the phase angle. As can be seen from the figure, the output power is mainly active power, while the reactive power is very small. Therefore, the reactive power compensation can be played indirectly by adjusting the phase angle of the output waveform.

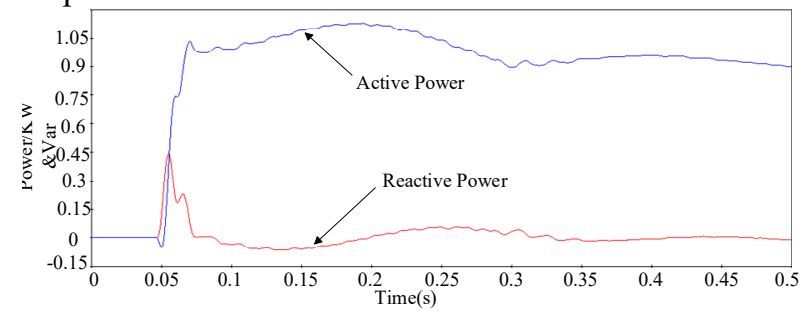

Fig.6. The active and reactive power of cascade H-bridge

\section{Conclusions}

In this paper, a new cascade H-bridge photovoltaic module structure is proposed, and the cascade H-bridge control strategy, inertial energy control strategy and power control strategy are analyzed, and the following conclusions are drawn:

(1) The cascade chain structure can replace the inverter and convert the direct current of the photovoltaic cell to alternating current. Using low-voltage switching devices to achieve high voltage output can eliminate the expense of inverter and reduce the cost of photovoltaic power station. When the photovoltaic cell is mismatched or the panel is blocked leading to the lack of chain, the cascade structure can still operate the $n-k$ lack of chain control technology through communication and distribution of the corresponding angle, which guarantees the improvement of the reliability of solar photovoltaic power generation.

(2) Inertial energy control can be realized by adding supercapacitor into each unit module of cascade $\mathrm{H}$ bridge photovoltaic module. Supercapacitor storage can suppress the output power fluctuations caused by light conditions or the passing of clouds.

\section{Acknowledgment}

The research in this paper was supported by Research on Energy Systems of Smart Park. Research and Demonstration on Key Technologies of Multi-functional Energy Router of Shanghai Science and Technology Commission (18DZ1203700).

\section{References}

1. Luo An, Xu Qianming, Ma Fujun, et al. Overview of power quality analysis and control technology for the smart grid. Journal of Modern Power Systems and Clean Energy, 4(1), 1-9. (2016)

2. Guan Mingyuan, Liu Qiang, Wu Guoqiang, et al. Smart photovoltaic integration system based on module cascade converter topology. High Voltage Engineering, 41(10), 3400-3406. (2015)

3. Zhang Song, Li Ying, Liang Wei, et al. Research on the converter control method based on the virtual synchronous generator technology. Electrical Measurement \& Instrumentation, 33(7), 5578-5591. (2014)

4. Zeng Zheng, Shao Weihua, Ran Li, et al. Mathematical model and strategic energy storage selection of virtual synchronous generators. Automation of Electric Power Systems, 39(13), 2231. (2015)

5. Tu Chunming, Lan Zheng, Xiao Fan, et al. Study on Cascade H-bridge Photovoltaic Power Systems With Synchronous Generator Characteristics. Proceedings of the CSEE, 37(2), 433-443. (2017)

6. Li Dongdong, Zhu Qianwei, Lin Shunfu, et al. A Self-Adaptive Inertia and Damping Combination Control of VSG to Support Frequency Stability. IEEE Transactions on Energy Conversion, 32(1), 397-398. (2017)

7. Wei Yalong, Zhang Hui, Sun Kai, et al. Presynchronization Method of Virtual Synchronous Generator Using Virtual Power. Automation of Electric Power Systems, 62(11), 7009-7024. (2016)

8. Shi Rongliang, Zhang Xing, Liu Fang, et al. Control Strategy of Virtual Synchronous Generator for Improving Frequency Stability of Islanded Photovoltaic-Battery-Diesel Microgrid. Automation of Electric Power Systems, 40(22), 77-85. (2016)

9. Huang Lipei, Ma Yiwei, Sun Kai, et al. Control of High Efficiency Cascade Photovoltaic Gridconnected Inverter. High Voltage Engineering, 37(3), 680-686. (2011)

10. Yu Yifan, Konstantinou Georgios, Hredzak Branislav, et al. Power balance optimization of cascade H-bridge multilevel converters for largescale photovoltaic integration. IEEE Transactions on Power Electronics, 31(2), 1108-1120. (2016)

11. Zhao Tao, Zhang Xing, Mao Wang, et al. A control strategy for cascade H-bridge Photovoltaic grid connected inverter. Power Electronics, (12), 84-86. (2017) 
12. Wang Fusheng, Zhang Dehui, Dai Zhiqiang, et al. A Hybrid Control Scheme of Cascade H-Bridge Inverters for Grid-Connection Photovoltaic Systems. Transactions of China Electrotechnical Society, 31(s1),137-145. (2017) 